\title{
The limb deformation of the compound bow
}

\author{
M. Tiermas
}

Received: 24 February 2016/ Accepted: 3 July 2016/Published online: 11 July 2016

(C) The Author(s) 2016. This article is published with open access at Springerlink.com

\begin{abstract}
A model of the archery round-wheel compound bow with a more realistic limb deformation is introduced. The model is based on the former compound bow model of the author with the supposition that the elastic limb deformation is consistent with the Euler-Bernoulli equation. The Young's modulus of an example bow limb is determined. The model is compared to the experimental data and to the former model which uses the modified Hickman approximation for the bow limbs. The effects of the shape of the limb on the force-draw curve of the compound bow are also considered. The model presented here may be used when designing or adjusting the limbs of the compound bow. An approximation for the path of the limb tip for the straight limb of uniform bending stiffness for small deformations is also derived.
\end{abstract}

Keywords Compound bow - Elastic deformation Force-draw curve $\cdot$ Eccentric wheel

M. Tiermas $(\triangle)$

Department of Physics, University of Helsinki, Helsinki, Finland

e-mail: marko.tiermas@helsinki.fi

\section{Introduction}

The compound bow, which was invented by Claude Lapp in 1938 [1], has become a popular instrument among the bow hunters and sportsmen. Yet, the mechanical action of the compound bow has not been investigated until quite recently. In the earlier studies [2-5] concerning compound bows the limb of the bow has been treated as a straight rod, which bends only on one point, which is either at the bottom of the limb or at some distance from the limb bottom. This approximation is the motivation of this paper.

Hickman introduced a simple model of the traditional long bow in [6], where he also noticed that the limb bends in the arc of circle. If the length of the limb is $L$, he also showed that the path made by the limb tip is an arc of the circle whose radius is $3 L / 4$ and whose center is located at a distance of $3 L / 4$ from the tip of the undeflected limb. This idea combined with the Hooke's law for the force acting on the tip of the limb is the basis of his long bow model. The Hickman model is confirmed by Tuijn and Kooi [7], and its modified version is also included in the compound bow model by the author [5].

The Hickman model was overtaken by Kooi in [8], where the deformation of the limb of the bow with or without recurve is thoroughly investigated. As a result, the initial shape and also the possible length-dependent bending stiffness of the limb can be taken into account. This kind of elastic limb bending is more realistic and also offers a possibility for further 
generalizations. The aim of this paper is to insert this elastic bending in the former model of the compound bow presented in [5].

\section{Formulation of the problem}

At first, we shall follow the theory of the static deformation of the bow limb as introduced by Kooi [8]. Let us assume that the limbs of the compound bow are inextensible, they are symmetric as in $[5,8]$, and can be represented by an elastic line of zero thickness. Then it will be sufficient to consider the upper part of the bow only. Let $L_{t o t}$ be the total length of the limb measured from the riser end (the bottom) of the limb to the axle point along the limb. As a part of the limb is inside the modified part of the riser (the limb pocket), let $L$ be the length of the free (elastic) limb measured from the point where the limb touches the riser to the axle point along the limb. Let us assume that the part of the limb which is inside the limb pocket is straight and rigid. This rigid part of length $L_{t o t}-L$ is also seen in Fig. 1 as the line $G O$.

Let $O=(x(0), y(0))$ be the point where the free limb meets the limb pocket. Let us call the point $G$ in Fig. 1 as the bottom of the limb, whereas the point $O$ may be called as the bottom of the free limb. The

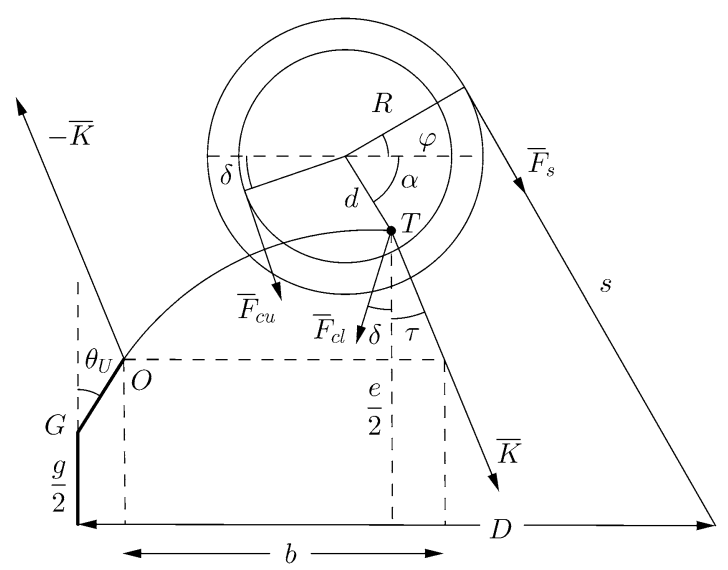

Fig. 1 The upper part of the compound bow in drawn position with related variables and forces. The size of the wheel system is exaggerated. In balance, the supporting force $-\bar{K}$ prevents the limb from moving. The force $\bar{K}$ acting on the limb tip $T$ is the sum of components $\bar{F}_{s}, \bar{F}_{c l}$ and $\bar{F}_{c u}$. The line segment $G O$ is the rigid part of the limb inside the limb pocket, whereas the curve $O T$ is the free limb. Note that the angle $\alpha$ is here negative eccentric system is fixed on the other end of the limb. Let us call the axle point $T=(x(L), y(L))$ of this eccentric system as the tip of the limb, as noted in Fig. 1. Let us also introduce the angle

$$
\lambda=\omega-\omega_{U}
$$

where $\omega$ is the angle of the infinitesimal segment of the limb with respect to the vertical line and $\omega_{U}$ the angle of the infinitesimal segment of the limb without the string (undeflected limb) with respect to the vertical line. There are also two geometric conditions,

$\frac{\mathrm{d} x}{\mathrm{~d} l}=\sin \left(\lambda+\omega_{U}\right)$

$\frac{\mathrm{d} y}{\mathrm{~d} l}=\cos \left(\lambda+\omega_{U}\right)$

where $x$ and $y$ are the Cartesian $\mathrm{x}$ - and $\mathrm{y}$-coordinates of the infinitesimal segment of the limb measured from the bottom of the free limb towards the tip of the limb, and $l$ the length coordinate along the limb measured from the bottom of the free limb with length $L$ towards the tip of the limb. The boundary conditions at $l=0$ are

$\lambda(0)=x(0)=y(0)=0$

Let us assume the Euler-Bernoulli equation for the elastic line is valid. With the help of geometrics and the Euler-Bernoulli equation, the following equation can be derived,

$W \frac{\mathrm{d} \lambda}{\mathrm{d} l}=K((b-x) \cos \tau-y \sin \tau)$

where $W$ is the bending stiffness of the limb, $K$ the absolute value of the total force $\bar{K}$ acting on the tip of the limb, $\tau$ the angle between $\bar{K}$ and the vertical line, and $b$ the distance between the bottom of the free limb and the point where the line that goes via the tip of the limb and is parallel to $\bar{K}$ meets the vertical line that goes via the bottom of the free limb. For the details of Eqs. (1)-(5), the reader is kindly asked to examine the paper [8], where the deformation of the conventional bow (with or without recurve) is presented elaborately.

Now our problem differs from [8], for the unknowns in the group of Eqs. (1)-(5) are $K$ and $b$. Also we must find $\tau$ and the respective limb tip coordinates $y_{L}=y(L)$ and $x_{L}=x(L)$.

In [5], it was supposed that the limb bends as in Hickman model with a slight modification, which is 
quite different case as here. However, we can still use the compound bow model introduced in [5] in order to find the limb tip y-coordinate and the angle $\tau$, for these values can be calculated without the Hickman assumption. From Fig. 1 we find that the y-coordinate of the limb tip with respect to the bottom of the free limb is

$y_{R}=\frac{e}{2}-\left(\frac{g}{2}+\left(L_{t o t}-L\right) \cos \theta_{U}\right)$

where $e$ is the distance between the axle points of the upper and the lower limbs, $g$ the length of the riser measured from the bottom of the upper limb to the bottom of the lower limb, and $\theta_{U}$ the angle between the vertical line and the undeflected straight part of the limb inside the limb pocket.

Hence, the solution of the group of Eqs. (1)-(5) must satisfy the equation

$y_{R}=y_{L}-y(0)$

where $y_{L}$ is the limb tip y-coordinate of the solution of the group of Eqs. (1)-(5). For the limb tip x-coordinate $x_{L}$ of the solution, there is another condition,

$\tan \tau=\frac{b-\left(x_{L}-x(0)\right)}{y_{R}}$

The equation for the absolute value of the total force $K$ acting on the tip of the limb can be derived from its Cartesian components, so according to Fig. 1,

$$
K^{2}=\left(F_{s} \sin \varphi\right)^{2}+\left(F_{s} \cos \varphi+2 F_{c} \cos \delta\right)^{2}
$$

where $F_{s}$ is the absolute value of the string tension, $\varphi$ the angle between the upper half of the string and vertical line and $\delta$ the angle between the horizontal line and the line that connects the centre of the upper wheel and the point where the straight cable contacts the upper cable (inner) eccentric. As in [5], the absolute value of the cable tension is

$$
F_{c}=\frac{d_{s}}{d_{c}} F_{s}
$$

where $d_{s}$ is the lever arm of the string tension and $d_{c}$ the lever arm of the cable tension. The angle between $\bar{K}$ and the vertical line is $\tau$, so from Eqs. (9) and (10) we see that

$$
\tan \tau=\frac{d_{c} \sin \varphi}{d_{c} \cos \varphi+2 d_{s} \cos \delta}
$$

\section{Numerical solution}

The angle $\tau$ can be calculated from Eq. (11) and $y_{R}$ from Eq. (6) with the help of the compound bow model presented in [5]. Let us suppose the bending stiffness $W(l)$ and the profile of the undeflected limb $\omega_{U}(l)$ are known. The first aim is to compute the values of $x_{L}$ and $y_{L}$. Using the initial guess values

$$
\widetilde{K}=580, \widetilde{b}=L \sin \theta_{U}
$$

we can solve the group of Eqs. (1)-(5) numerically with the classical Runge-Kutta (RK) method. The resulting estimates of the RK method for the Cartesian limb tip coordinates are shortly expressed as

$$
\left(\widetilde{x}_{L}, \widetilde{y}_{L}\right)=f_{R K}(\widetilde{K}, \widetilde{b}, l=L)
$$

These estimates are also needed when computing the zeros of the two following functions based on condition Eqs. (7) and (8),

$f_{1}(\widetilde{K}, \widetilde{b})=y_{R}-\left(\widetilde{y}_{L}-y(0)\right)=0$

$f_{2}(\widetilde{K}, \widetilde{b})=\frac{\widetilde{b}-\left(\widetilde{x}_{L}-x(0)\right)}{y_{R}}-\tan \tau=0$

The problem is now to find the simultaneous zeros of these two functions. The group of Eqs. (13)-(15) including the RK method was solved with the trustregion dogleg method [9]. After solution, the resulting values of $K$ and $b$ were set again into the RK algorithm, when the final values of the limb tip coordinates $x_{L}$ and $y_{L}$ could be computed straightforwardly.

With the help of Fig. 1 the draw, which is here defined as the distance between the midpoint of the string and the vertical line that connects the bottoms of the upper and the lower limb, can now be expressed as $D=\left(L_{t o t}-L\right) \sin \theta_{U}+x_{L}-d \cos \alpha+R \cos \varphi+s \sin \varphi$

where $d$ is the distance between the axle and the centre of the wheel, $R$ the radius of the string (outer) eccentric, $\alpha$ the angle between the horizontal line and the line that connects the centre of the upper wheel and the upper axle point, and $s$ the length of the straight half-string. The string tension can be solved from Eqs. (9) and (10), 


$$
F_{s}=\frac{K d_{c}}{\sqrt{d_{c}^{2}+4 d_{s} d_{c} \cos \varphi \cos \delta+4 d_{s}^{2} \cos ^{2} \delta}}
$$

Due to symmetry, the absolute value of the force acting on the arrow is, according to Fig. 1,

$F=2 F_{s} \sin \varphi$

which completes our treatment.

\section{Results of model testing}

The same bow as presented in [5] was selected for model testing. From this bow, the length of the limb part which is inside the limb pocket was measured with the steel ruler, resulting in the value of $L_{t o t}-L=8.5 \mathrm{~cm}$. The other initial parameters of the bow are presented in Table 1 of paper [5] and are not repeated here. Note that in [5] parameter $L$ denotes the total limb length, which is in this paper $L_{t o t}$. In addition of these parameters, the bending stiffness of the bow limb is needed. In order to determine it, let us assume that the limb material is homogeneous, and the cross-section of the limb is a rectangle. Let $E$ be the Young's modulus of the limb material, and let us also assume that $E$ is constant. The bending stiffness of the limb is then

$$
W(l)=E I(l)
$$

where $I(l)$ is the second moment of inertia of the limb. If the centroid of the cross-section rectangle is located at the origin, the height $h(l)$ of this rectangle (limb thickness) parallel to y-axis and the width $w(l)$ parallel to $\mathrm{z}$-axis, the second moment of inertia at $l$ with respect to the $\mathrm{z}$-axis is [11]

$I(l)=\frac{w(l) h^{3}(l)}{12}$

The width and the thickness of the limb with several $l$ coordinates were measured with the calipers. The cubic splines were fitted for both the width and the thickness data of the limb for continuity reasons. The second moment of inertia with respect to the limb length coordinate is presented in Fig. 2.

The Young's modulus $E$ of the limb material was determined indirectly with the help of the model. First, 500 evenly distributed values of the prime variable $\alpha$ were selected from the domain $-194^{\circ} \leq \alpha \leq 52.5^{\circ}$.

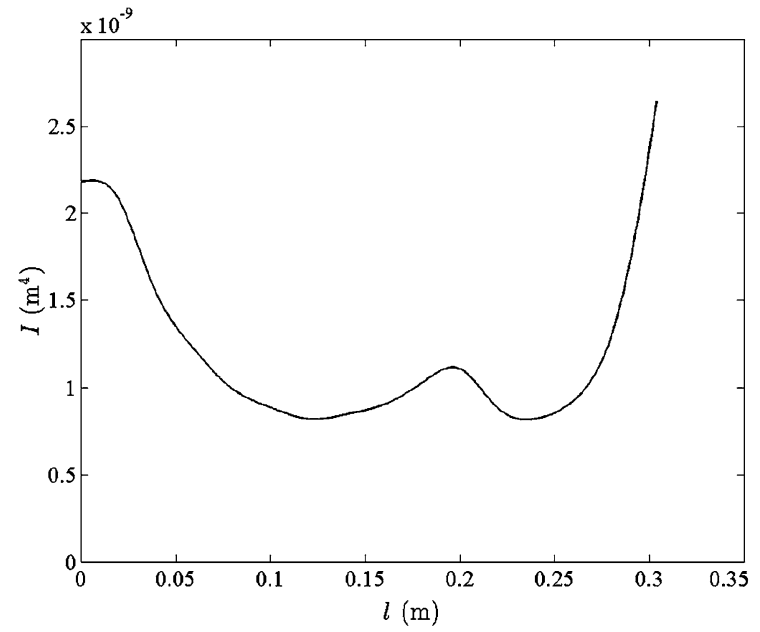

Fig. 2 The second moment of inertia of the measured limb as the function of limb length coordinate $l$

Using an initial guess value for $E$ and the other initial parameter values of Table 1 of paper [5], the procedure described in Sect. 3 was executed separately with every value of $\alpha$, resulting also the respective values of $D$ and $F$.

Finally, the value of $E$ was chosen so that the model fits to the measurements as well as possible in the sense of least squares. For continuity reasons, a cubic spline was fitted to the $(D, F)$-values of the model, and the least squares method was applied on the values of this spline and the measured data. Both the drawing and the relaxing FD data of the bow were included in the fitting. With the value of $E=33.75 \mathrm{GPa}$ the sum of the squares is least, so this value of $E$ can be considered to be the best estimate.

With this value of $E$ the model match on the FD data is good. The figure about the resulting FD curve of the model and the measured FD data is visually not separable from Fig. 7 of paper [5], so it is not presented here. With the parameter values mentioned before, from the initial position to full draw the height difference between the FD curve of this model and the model of [5] is $<0.8 \mathrm{~N}$. The differences between the models for the placement of the limb tip and for the draw are also very small, both being $<0.3 \mathrm{~mm}$.

The potential energy of the bow can be calculated from the FD curve of the model, it is

$$
V=\int_{D_{0}}^{D_{F}} F(D) \mathrm{d} D
$$


where $D_{F}$ denotes the full draw and $D_{0}$ the draw in the initial position. On the other hand, Eq. (5) describes also the bending moment,

$$
M(l)=W \frac{\mathrm{d} \lambda}{\mathrm{d} l}=W\left(\frac{\mathrm{d} \omega}{\mathrm{d} l}-\frac{\mathrm{d} \omega_{U}}{\mathrm{~d} l}\right)
$$

so from the initial position to the full draw, the potential energy stored to the limbs is

$V=\left[\int_{0}^{L} W(l)\left(\omega^{\prime}(l)-\omega_{U}^{\prime}(l)\right)^{2} \mathrm{~d} l\right]_{D=D_{0}}^{D=D_{F}}$

which can be used for checking the computations. Using the above mentioned parameter values, the cubic spline functions were fitted to the $(D, F),(l, W)$, $(l, \omega)$ and $\left(l, \omega_{U}\right)$ value pairs of the model for continuity reasons. After differentiating, the cubic spline functions were also fitted to the $\left(l, \omega^{\prime}\right)$ and $\left(l, \omega_{U}^{\prime}\right)$ value pairs, when the integrations of the equations (21) and (23) were possible. Using $500 \mathrm{knot}$ points the calculations of Eqs. (21) and (23) differed $<0.15 \%$.

Another check was made selecting the bending stiffness as constant $\left(W_{0}\right)$ and the limbs straight and in vertical position when undeflected. Choosing $L_{t o t}=L$ and taking the other relevant initial parameters from the bow presented in [5], the method explained before gives us a solution including the values for $K, b$ and $x_{L}$. However, in order to avoid numerical problems with RK method, the values $\omega_{U}(l)=0.01^{\circ}$ were selected for the initial angle instead of $\omega_{U}(l)=0$. Considering the situation at the initial position $\tau=0$, when according to [10] the energy of the limbs can also be expressed as

$$
V_{0}=4 \sqrt{K W_{0}}\left(E_{2}(p)-\left(1-p^{2}\right) E_{1}(p)\right)
$$

where $E_{1}$ and $E_{2}$ are the complete elliptic integrals of the first and the second kind, and

$p=\sin (\omega(L) / 2)$

Varying the parameter $e_{0}$ in the domain $0.80 \mathrm{~m}$ $\leq e_{0} \leq 1.10 \mathrm{~m}$, several initial position energies were calculated using 500 knot points. The integral in brackets in Eq. (23) can be used to compute the potential energy also in the initial position. The differences between the calculations based on Eqs. (24) and (23) were $<0.1 \%$.

\section{Other results}

The model presented here gives us a possibility to consider the effects of the material, shape and dimensions of the limb on the FD curve of the compound bow. The bending stiffness of the limb is determined by the dimensions of the limb and the Young's modulus $E$ of the limb material. From Eq. (19) we notice that the effect of $E$ on the stiffness function is linear, so varying $E$ only the height scale changes but the shape of the FD curve remains the same. The width and the thickness of the limb can also be varied in numberless ways. Both these dimensions determine the second moment of inertia $I(l)$ of the limb, but the variations in thickness has more crucial effects on bending stiffness due to the third power relation of Eq. (20).

It was observed that with too small values of $W(l)$ serious computational problems may arise. The same effect is reported also in [8]. For this reason it was checked that

$$
W(l) \geq 1.40 \mathrm{Nm}^{2}, \quad 0 \leq l \leq L
$$

whenever manipulating the second moment of inertia $I(l)$ or the Young's modulus $E$.

With a straight undeflected limb, it turned out that also the second moment of inertia $I(l)$ affects mainly on the height of the FD curve. The changes on the shape and on the width of the FD curve are minimal. The effect on the width of the FD curve is greatest when $I(l)$ is chosen so that it has a clear minimum point quite near on the tip of the limb. Then we have almost a "hinge" at the respective point of the limb, when the full draw is clearly shortened from original.

In order to study the effect of the limb side profile, some parameters of the bow were selected as constants. The fixed parameters were $g=38.1 \mathrm{~cm}$, $L=30.4 \mathrm{~cm}, \quad L_{t o t}=38.9 \mathrm{~cm}, \quad e_{0}=87.0 \mathrm{~cm}$, $\alpha_{0}=52.5^{\circ}, R=5.36 \mathrm{~cm}, r=3.98 \mathrm{~cm}, d=2.74$ $\mathrm{cm}, W(l)=W_{0}=18.0 \mathrm{Nm}^{2}, \theta_{U}=\omega_{U}(0)=25.0^{\circ}$. Three different limb side profiles were created. These limb profiles in undeflected, initial and full draw positions are presented in Fig. 3. 


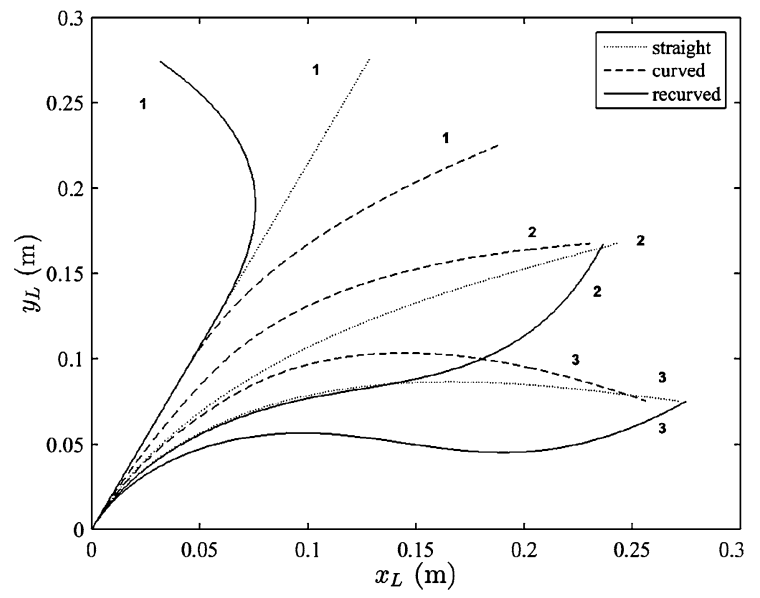

Fig. 3 The side profiles of the straight, curved and recurved upper limb in undeflected (1), initial (2) and full draw (3) positions

As can be seen from Fig. 3, the displacement of the limb tip from undeflected to the initial position is least for the curved and greatest for the recurved limb. This was due to the fixed values of $e_{0}$ and $\omega_{U}(0)$. When the bending stiffness is the same for all three limbs, this implies that the bending tension is greatest for the recurved limb, so the peak of the FD curve with the recurved limbs will be highest and with the curved limbs lowest. When comparing the FD curves of these limbs, it is therefore reasonable to use a dimensionless knock-down factor to the force values for normalization. Alternatively we could manipulate the bending stiffness of the limbs of different profiles so that the peak forces are the same. The normalized FD curves of the compound bows with these three limb systems with knock-down values 1.0 for the straight limb, 1.611 for curved limb and 0.654 for the recurved limb are presented in Fig. 4.

From Fig. 4 we notice that compared to other FD curves, with curved limbs the slope of the front part of the FD curve is least and the force in full draw greatest. Otherwise the differences between the normalized FD curves are rather small. This is somewhat surprising because in case of traditional recurve bows, the shape of the FD curve can be strongly affected by changing the shape of the limb profile, as demonstrated in [8]. The crucial difference is that on a recurve bow the end parts of the string are twisted along the limbs on the archer's side in brace height situation. When the recurve bow is drawn, the length of the acting string changes all the time, likewise the point where the

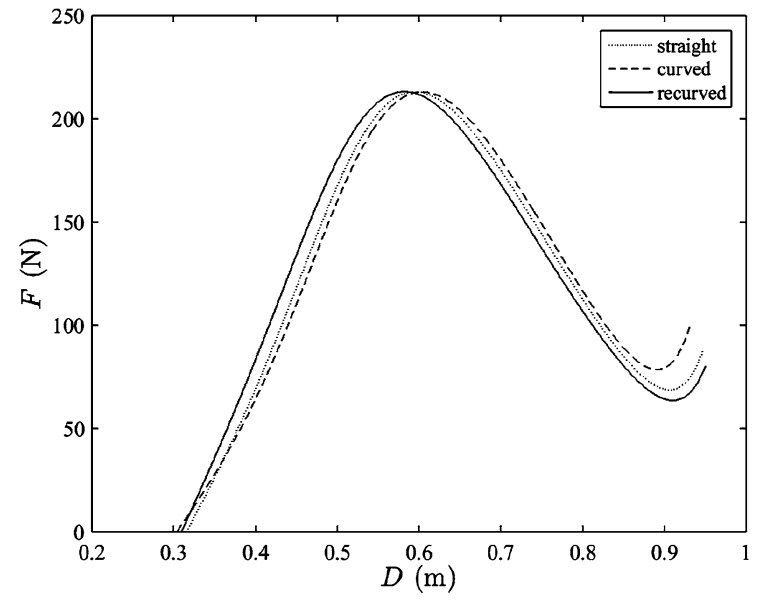

Fig. 4 The normalized calculated FD curves of the compound bow with the straight, curved and recurved limbs

string touches the limb. Thus also the length of the acting limb changes, and finally the FD curve of the recurve bow is quite unique, as presented in [8]. In compound bows the point where the string and the cable forces act to the limb is the axle point (or the tip of the limb, as assumed here), so the acting limb length remains constant. Also there is a slot in the limbs for the eccentric systems, so the cables may freely go through the limbs, when the line parallel to the total force vector acting to the limb tip may also intersect the recurved limb line. Unlike with traditional recurve bows, with too excessive recurved compound bow limbs the tips of the limbs may work in impractical "wrong" direction, when also buckling is possible.

For straight limbs with constant width and thickness, for small deformations the path made by the tip of the (free) limb of length $L$ can be approximated by an arc of a circle whose radius is $5 L / 6$ and whose center is located at a distance of $5 L / 6$ from the tip of the undeflected limb. Hence we have an estimate to parameter $A$ used in [5],

$A=\frac{5}{6} \frac{L}{L_{t o t}}$

Also the approximation for the spring constant of the elastic portion of the limb is then

$k=\frac{15 W_{0}}{6 L^{2}}$

These results are derived in "Appendix". It is noteworthy that using the supposition of straight limbs with constant bending stiffness, the values $L=30.4$ 
$\mathrm{cm}$ and $L_{\text {tot }}=38.9 \mathrm{~cm}$, and the parameter values of Table 1 of paper [5] with the estimates of Eqs. (27) and (28) for $A$ and $k$, the differences between the FD curves of this model and the model of paper [5] were visually observable mainly on the peak force area, where the height differences were still $<1 \%$.

\section{Conclusion}

The more realistic limb deformation according to Euler-Bernoulli (EB) equation was inserted to the former model of [5]. The second moment of inertia of the compound bow limb was measured, and the Young's modulus $E$ of the limb material was determined by curve fitting. The resulting FD curve of the model fits very well on measured data. Also both the calculated FD curve and the calculated path of the limb tip differed only very slightly when compared to calculations with the earlier model of [5], which uses the modified Hickman $(\mathrm{H})$ model for the limb deformation. In case of straight (undeflected) compound bow limbs with constant bending stiffness, there is no significant difference whether we use EB model or the modified $\mathrm{H}$ model for the deformation of the limb.

An approximation for the path of the tip of the straight limb with constant bending stiffness for small deformations was derived. Then using the modified $\mathrm{H}$ model the measurement of $A$ is no longer an absolute necessity, and $k$ can also be estimated from the bending stiffness of the limb.

The effects of varying the thickness, width and side profile of the limb were considered. It was found that in all cases mainly the height of the FD curve and the full draw changed, when the general shape of the curve remained rather unchanged.

When modelling the compound bow, both the EB model introduced in this paper and the earlier modified $\mathrm{H}$ model can be used for the deformation of the limb. If the limbs differ clearly from straight rods of constant width and thickness when undeflected, EB model is recommended. In bow design the EB model is also superior, for then only the dimensions and the shape of the limbs with the Young's modulus of the limb material are needed, and there is no need to evaluate the parameters $A$ and $k$ at all. While computationally more demanding, the model with the EB limb deformation is also more accurate and can be more helpful when adjusting the compound bow limb or choosing the limb material. Finally, the reader is reminded that considerations presented here are based on statics only.

\section{Appendix: The path of the tip of the straight limb with constant bending stiffness}

In modern compound bow the limbs are sometimes made of two straight rods with even or almost even width and thickness. Let us assume that the point of the tip of the limb is $(0,0)$ and the undeflected straight limb is parallel to positive $\mathrm{x}$-axis. We also suppose that the force $K$ acting on the limb tip is perpendicular with respect to the limb. If the material of the limb is homogeneous and the Young's modulus $E$ of the limb is constant, the curvature of the limb for small deformations is according to Euler-Bernoulli equation

$\frac{1}{\rho}=\frac{\mathrm{d}^{2} y}{\mathrm{~d} l^{2}}=\frac{K l}{E I_{0}}$

where $\rho$ is the radius of the curvature and $I_{0}$ the second moment of inertia of the limb. Note that the coordinate $l$ is here measured along the limb starting from the tip of the limb. We notice that the curvature increases linearly towards the bottom of the limb, so the shape of the limb is a part of a spiral, for the curvature for the Cornu spiral is

$\frac{1}{\rho}=\frac{l}{c^{2}}$

where $c$ is a parameter, and the Cartesian coordinates of this spiral can be expressed with the help of Fresnel integrals as

$x(l)=\int_{0}^{l} \cos \frac{t^{2}}{2 c^{2}} \mathrm{~d} t, \quad y(l)=\int_{0}^{l} \sin \frac{t^{2}}{2 c^{2}} \mathrm{~d} t$

where $l$ is the length of the spiral arc [12]. It will be a bit more convenient to replace the parameter $c$ with

$a=\frac{1}{c \sqrt{2}}$

when the angle of the tangent line at the point $l=L$ can be expressed [12] as

$$
\Psi=a^{2} L^{2}
$$

After a variable change in Eq. (31) we get the coordinates of the bottom of the free (elastic) limb, 
$x_{B}=\frac{1}{a} \int_{0}^{a L} \cos x^{2} \mathrm{~d} x, \quad y_{B}=\frac{1}{a} \int_{0}^{a L} \sin x^{2} \mathrm{~d} x$

From Eqs. (29), (30) and (32) we get the value

$$
a=\sqrt{\frac{K}{2 E I_{0}}}
$$

so the respective Cartesian coordinates $\left(x_{B}, y_{B}\right)$ can be calculated. The integrals of Eq. (34) cannot be solved analytically. However, by using Taylor series for sine and cosine we may write these integrals as

$$
\begin{aligned}
& \int_{0}^{a L} \cos x^{2} \mathrm{~d} x=\sum_{n=0}^{\infty}(-1)^{n} \frac{(a L)^{4 n+1}}{(2 n) !(4 n+1)} \\
& \int_{0}^{a L} \sin x^{2} \mathrm{~d} x=\sum_{n=0}^{\infty}(-1)^{n} \frac{(a L)^{4 n+3}}{(2 n+1) !(4 n+3)}
\end{aligned}
$$

Now we assume that $\left(x_{H}, y_{H}\right)$ is the hinge point of the undeflected limb from where we can bend the straight rigid limb in such a way that the coordinates of the bottom of the free limb will satisfy Eqs. (34). The path of the tip is then on the arc of the circle, which center is at the point $\left(x_{H}, y_{H}\right)$ and radius is some constant $C$ as illustrated in Fig. 5.

The length of the limb is unchanged, so the distance of the point $\left(x_{H}, y_{H}\right)$ from the bottom of the free limb is

$$
B=L-C
$$

From Fig. 5 we may write the condition equations

$$
\begin{aligned}
& \left(x_{B}-x_{H}\right)^{2}+\left(y_{B}-y_{H}\right)^{2}=B^{2} \\
& C^{2}=x_{H}^{2}+y_{H}^{2}
\end{aligned}
$$

There is one more condition, for the point $\left(x_{H}, y_{H}\right)$ is on the tangential line of the end point $\left(x_{B}, y_{B}\right)$ of our spiral. The slope of the spiral at the point $\left(x_{B}, y_{B}\right)$ is then

$$
m=\tan \Psi=\tan \left(a^{2} L^{2}\right)=\frac{y_{B}-y_{H}}{x_{B}-x_{H}}
$$

From (39) we get with the help of Eq. (41)

$$
\left(1+m^{2}\right)\left(x_{B}-x_{H}\right)^{2}=B^{2}
$$

and from (40) using Eqs. (38), (39), (41) and (42)

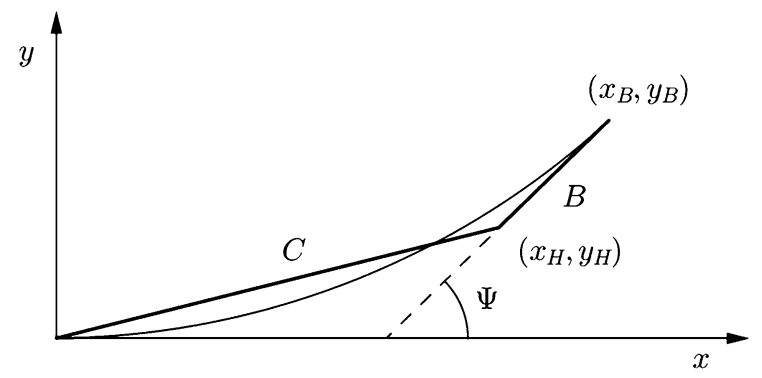

Fig. 5 The EB model (curve) and the modified $\mathrm{H}$ model of the deflected limb. In the modified $\mathrm{H}$ model, the hinge point $\left(x_{H}, y_{H}\right)$ divides the limb in two rigid parts of length $C$ and $B$. The hinge point is on the tangential line (dashed line) of the end point $\left(x_{B}, y_{B}\right)$ of the curve. Note that here the tip of the limb is in the point $(0,0)$ and the point $\left(x_{B}, y_{B}\right)$ is the bottom of the free limb

$$
\begin{array}{r}
\left(L-\left(x_{B}-x_{H}\right) \sqrt{1+m^{2}}\right)^{2}=x_{H}^{2}+y_{B}^{2} \\
-2 y_{B} m\left(x_{B}-x_{H}\right)+m^{2}\left(x_{B}-x_{H}\right)^{2}
\end{array}
$$

from which after some manipulation we get

$$
x_{H}=x_{B}+\frac{y_{B}^{2}+x_{B}^{2}-L^{2}}{2 L \sqrt{1+m^{2}}-2 x_{B}-2 y_{B} m}
$$

when Eq. (42) can be written as

$$
B=\sqrt{1+m^{2}} \frac{L^{2}-y_{B}^{2}-x_{B}^{2}}{2 L \sqrt{1+m^{2}}-2 x_{B}-2 y_{B} m}
$$

Using Eqs. (34), (36), (37), the Taylor series of the square root and substituting the slope $m$ with Taylor series for tangent, for small deformations we have from Eq. (45) after simplifying

$\lim _{a \rightarrow 0} B=\frac{1}{6} L$

so for the radius

$\lim _{a \rightarrow 0} C=\lim _{a \rightarrow 0}(L-B)=\frac{5}{6} L$

Thus for a straight even-wide limb of length $L$, the path made by the limb tip for small deformations is an arc of the circle whose radius is $C=5 L / 6$ and whose center is located at a distance of $5 L / 6$ from the tip of the undeflected limb. In this derivation we have assumed that the limb can deflect from the tip to the bottom of the free limb. So if we want to use this approximation in the model of [5], the constant $A$ can be estimated as 


$$
A=\frac{C}{L_{\text {tot }}}
$$

where $L_{\text {tot }}$ is the limb length including the part inside the limb pocket. Also the constant $k$ in paper [5] can now be estimated with the help of the Young's modulus $E$ of the limb material and the dimensions of the limb. Considering now the point $\left(x_{B}, y_{B}\right)$ as origin, the coordinate $l=0$ at origin and $l=L$ at the tip of the limb, and the line perpendicular to line $B$ in Fig. 5 as y-axis, Eq. (29) may be written as

$$
\frac{\mathrm{d}^{2} y}{\mathrm{~d} l^{2}}=\frac{K(L-l)}{E I_{0}}
$$

With the assumptions mentioned before, for small deformations after integrating Eq. (49) we have

$$
y(L)=\frac{K L^{3}}{3 E I_{0}}
$$

The deflection $y(L)$ can be expressed with the help of limb deflection angle $\Delta \theta$,

$$
y(L)=C \Delta \theta
$$

so the constant $k$ for the bending portion of the limb is

$$
k=\frac{K}{\Delta \theta}=\frac{3 E I_{0} C}{L^{3}}=\frac{15 E I_{0}}{6 L^{2}}
$$

Acknowledgments This research was financially supported by Byro Energiatekniikka Oy.

Open Access This article is distributed under the terms of the Creative Commons Attribution 4.0 International License (http:// creativecommons.org/licenses/by/4.0/), which permits unrestricted use, distribution, and reproduction in any medium, provided you give appropriate credit to the original author(s) and the source, provide a link to the Creative Commons license, and indicate if changes were made.

\section{References}

1. Aronson RB (1977) The compound bow: ugly but effective. Mach Des 10(25):38-40

2. Park JL (2009) A compound archery bow dynamic model, suggesting modifications to improve accuracy. Proc Inst Mech Eng Part P J Sports Eng Technol 223:139-150

3. Park JL (2009) Compound archery bow nocking point locus in the vertical plane. Proc Inst Mech Eng Part P J Sports Eng Technol 224:141-154

4. Zanevskyy IP (2012) Compound archery bow asymmetry in the vertical plane. Sports Eng 15:167-175

5. Tiermas M (2015) An advanced model of the round-wheel compound bow. Meccanica. doi:10.1007/s11012-0150262-5

6. Hickman CN (1937) The dynamics of bow and arrow. J Appl Phys 8:404-409

7. Tuijn C, Kooi BW (1992) The measurement of arrow velocities and the efficiency of working-recurve bows. Eur J Phys 13:127-134

8. Kooi BW (1980) On the static deformation of the bow. J Eng Math 14(1):27-45

9. Moré JJ, Garbow BS, Hillstrom KE (1980) User guide for MINPACK 1. Argonne National Laboratory, Rept. ANL80-74

10. Frisch-Fay R (1962) Flexible bars. Butterworth, London

11. Kinasoshvili R (1978) Strength of materials. Mir Publishers, Moscow

12. Kreyszig E (1959) Differential geometry. University of Toronto Press, Toronto 